\title{
Applications of Boolean Algebra: Claude Shannon and Circuit Design
}

\author{
Janet Heine Barnett*
}

26 January 2009

\section{Introduction}

On virtually the same day in 1847, two major new works on logic were published by prominent British mathematicians: Formal Logic by Augustus De Morgan (1806-1871) and The Mathematical Analysis of Logic by George Boole (1815-1864). Both authors sought to stretch the boundaries of traditional logic by developing a general method for representing and manipulating logically valid inferences or, as DeMorgan explained in an 1847 letter to Boole, to develop 'mechanical modes of making transitions, with a notation which represents our head work' [18, p. 25 ]. In contrast to De Morgan, however, Boole took the significant step of explicitly adopting algebraic methods for this purpose. As De Morgan himself later proclaimed, "Mr. Boole's generalization of the forms of logic is by far the boldest and most original ..." (as quoted in [13, p. 174]).

Boole further developed his bold and original approach to logic in his 1854 publication An Investigation of the Laws of Thought ${ }^{1}$. In this work, Boole developed a system of symbols $(\times,+)$ representing operations on classes (or sets) which were symbolically represented by letters. In essence, his logical multiplication $x y$ corresponded to today's operation of set intersection, and his logical addition $x+y$ to today's operation of set union. ${ }^{2}$ Using these definitions, Boole then developed the laws of this 'Algebra of Logic,' many of which also held true in 'standard algebra'. Other laws, however, differed substantially from those of standard algebra, such as the Idempotent Law ${ }^{3}: x^{2}=x$.

As noted by Boole, the Idempotent Law holds in standard algebra only when $x=0$ or $x=1$. He further commented $[4$, p. 47] that for

... an Algebra in which the symbols $x, y, z$, \&c. admit indifferently of the values 0 and 1 , and of these values alone ... the laws, the axioms, and the processes ... will be identical in their whole extent with the laws, the axioms, and the processes of an Algebra of Logic.

\footnotetext{
${ }^{*}$ Department of Mathematics and Physics; Colorado State University-Pueblo; Pueblo, CO 81001 - 4901 ; janet.barnett@colostate-pueblo.edu.

${ }^{1}$ For further details on Boole's work in logic and modifications made to it by John Venn (1834-1923) and C. S. Peirce (1839-1914), see the project "Origins of Boolean Algebra in the Logic of Classes: George Boole, John Venn and C. S. Peirce," Janet Barnett author.

${ }^{2}$ For various technical reasons, Boole restricted his use of + to classes which were disjoint. Most of his immediate followers, however, relaxed this restriction, so that their use of + corresponded exactly to today's operation of set union. British mathematician John Venn (1834-1923) discussed this issue in detail in the second (1894) edition of his Symbolic Logic [20, pp. 42-46]. Ultimately, Venn adopted an unrestricted use of + 'partly ... because the voting has gone this way, and in a matter of procedure there are reasons for not standing out against such a verdict ...'.

${ }^{3}$ For Boole, the Idempotent Law followed directly from the definition of $x y$ as 'the whole of that class of objects to which the names or qualities represented by $x$ and $y$ are together applicable ', from which 'it follows that if the two symbols have exactly the same signification, their combination expresses no more than either of the symbols taken alone would do.' (See [4, p. 31].) Selecting the sheep from the class of sheep, for instance, gives us just the class of sheep, so that $x x=x$.
} 
In the early twentieth century, this special two-valued 'arithmetical algebra' became important in the axiomatization of boolean algebras; Edward V. Huntington, for example, employed it as a model for one of three postulate sets for boolean algebra in his 1904 paper Sets of Independent Postulates for the Algebra of Logic ${ }^{4}$. In that work, Huntington defined addition and multiplication (which he denoted by $\oplus$ and $\odot$ respectively) by the following tables [10, p. 293]:

\begin{tabular}{c|ll}
$\oplus$ & 0 & 1 \\
\hline 0 & 0 & 1 \\
1 & 1 & 1
\end{tabular}

\begin{tabular}{c|ll}
$\odot$ & 0 & 1 \\
\hline 0 & 0 & 0 \\
1 & 0 & 1
\end{tabular}

For Huntington, these tables defined a completely abstract (i.e., meaningless) system. For Boole, the equalities represented in these tables (e.g., $1+1=1)^{5}$ would have represented statements about sets (i.e., the union of the universal set with itself is again the universal set). In this project, we will see how this same two-valued system was employed in another concrete application of boolean algebra in the mid-twentieth century: the design and analysis of circuits.

\section{Claude Shannon, Boolean Algebra and Circuit Design}

The algebraic methods introduced by Boole for the study of logic attracted considerable attention from mathematicians in the years following publication of Laws of Thought. Alongside the various refinements and extensions made to Boole's system during these years, mathematics itself underwent significant changes, becoming both increasingly abstract and more formal in its approach to proof. In keeping with this trend, there came a loosening of the ties between the algebraic system introduced by Boole and logic as a concrete interpretation of that system. In his classic The Algebra of Logic of 1914, for example, French mathematician Louis Couturat (1868-1914) went so far as to declare [6, p. 1]:

The formal value of this calculus and its interest for the mathematician are absolutely independent of the interpretation given it and of the application which can be made of it to logical problem. In short, we shall discuss it not as logic but as algebra. ${ }^{6}$

\footnotetext{
${ }^{4}$ For further details on Huntington's work, see the project "Boolean Algebra as an Abstract Structure: Edward V. Huntington and Axiomatization," Janet Barnett author.

${ }^{5}$ In Boole's 'Algebra of Logic', the symbols ' 0 ' and ' 1 ' denoted two special classes: 'nothing' ('empty set') and 'universe' ('universal set') respectively. To justify the use of these symbols, Boole used the analogy between the roles played by these numbers in algebra and the roles played by these special classes in logic [4, p. 47-48]. He argued, for example, that since $0 y=0$ in standard algebra, then '. . we must assign to the symbol 0 such an interpretation that the class represented by $0 y$ may be identical with the class represented by 0 , whatever the class $y$ may be. A little consideration will show that this condition is satisfied if the symbol 0 represent Nothing.' A similar analysis of the algebraic equation $1 y=y$ led him to conclude that ". . the class represented by 1 must be "the Universe," since this is the only class in which are found all the individuals that exist in any class.'

${ }^{6}$ Similarly, Huntington opened his 1904 paper with the following declaration [10, p. 288]: 'The algebra of symbolic logic, as developed by Leibniz, Boole, C.S. Peirce, E. SchröDer, and others is described by Whitehead as "the only known member of the non-numerical genus of universal algebra." This algebra, although originally studied merely as a means of handling certain problems in the logic of classes and the logic of propositions, has recently assumed some importance as an independent calculus; it may therefore be not without interest to consider it from a purely mathematical or abstract point of view, and to show how the whole algebra, in its abstract form, may be developed from a selected set of fundamental propositions, or postulates, which shall be independent of each other, and from which all the other propositions of the algebra can be deduced by purely formal processes. In other words, we are to consider the construction of a purely deductive theory, without regard to its possible applications.'
} 
It was not long, however, before individuals interested in problems outside of mathematics proper gained exposure to boolean algebra and its unique properties, thanks in part to the work of Couturat and other mathematicians interested solely in its formal algebraic structure. A 1949 list of some of the applications which resulted from that exposure - applications largely undreamed of by Boole and his Victorian colleagues - included "an axiomatic formulation of biology, the study of neural networks in the nervous systems, the analysis of insurance policies, probability and set theory, etc. [16, p. 588]". The compiler of this list, American mathematician and electrical engineer Claude E. Shannon (1916-2001), himself gained reknown for a particular application of boolean algebra.

Shannon completed bachelor degrees in both mathematics and electrical engineering at the University of Michigan in 1936. Two years later, at the age of 22, he completed a master's thesis in electrical engineering at the Massachusetts Institute of Technology. The idea which inspired his thesis work came from his exposure to symbolic logic in an undergraduate philosophy course. Vannever Bush (1890-1974), dean of engineering at MIT and inventor an early mechanical computer called the differential analyser machine, was sufficiently impressed by Shannon's thesis to sponsor its publication in an engineering journal. This award-winning paper went on to revolutionize the study of switches and relays, which in turn form the circuitry behind the binary arithmetic of modern comput$\mathrm{ers}^{7}$. Shannon then completed a doctorate in mathematics at MIT with a thesis on the application of mathematics to genetics, and began his official career as a research mathematician at Bell Laboratories in 1941. His association with Bell Labs, either as full time scientist or as a consultant, continued until 1971. In 1948, he published yet another ground breaking paper, A Mathematical Theory of Communication, thereby launching the still flourishing field of information theory. He married in 1949 (he and his wife had four children), and served as a faculty member and researcher at MIT from 1956 through 1978. His work included important contributions to cryptography, game theory and computer science; Shannon is also remembered for various mechanical inventions, and for his successful stock investment strategies. Among his many honors was the first ever Marconi Lifetime Achievement Award, awarded to him in 2000. By this time, sadly, Shannon suffered significantly from the effects of Alzheimer's disease; he died in a nursing home just a year later.

In the 1938 paper based on his master's thesis, A Symbolic Analysis of Relay and Switching Circuits, Shannon described the general problem to be solved and his proposed approach to it as follows $[14$, p. 713$]$ :

\section{0}

In the control and protective circuits of complex electrical systems it is frequently necessary to make intricate interconnections of relay contacts and switches. Examples of these circuits occur in automatic telephone exchanges, industrial motor-control equipment, and in almost any circuits designed to perform complex operations automatically. In this paper a mathematical analysis of certain of the properties of such networks will be made. ...

The method of attack on these problems may be described briefly as follows: any circuit is represented by a set of equations, the terms of the equations corresponding to the various relays and switches in the circuit. A calculus is developed for manipulating these equations by simple mathematical processes, most of which are similar to ordinary algebraic algorisms. This calculus is shown to be exactly analogous to the calculus of propositions used in the symbolic study of logic.

\section{0}

\footnotetext{
${ }^{7}$ For more information on the connection of switch/relay circuitry to binary arithmetic, see the project "Arithmetic Backwards from Shannon to the Chinese Abacus," Jerry Lodder author.
} 
On one level, the key to applying symbolic boolean algebra to relay and switching circuits lay in the fact that there are only two possible states for such circuits, open and closed, a situation reminiscent of Boole's special algebra on two symbols, 0 and 1. In fact, the arithmetical version of Shannon's postulates for networks as stated in this paper [14, p. 713] is identical to Huntington's two-valued model of boolean algebra (see page 2 of this project):

1000000000

\section{Postulates}

1. a. $0 \cdot 0=0$

b. $1+1=1$

2. a. $1+0=0+1=1$

b. $0 \cdot 1=1 \cdot 0=0$

3. a. $0+0=0$

b. $1 \cdot 1=1$

4. At any given time either $X=0$ or $X=1$.

10000000000

In a 1987 interview $^{8}$ with Omni magazine, Shannon elaborated on the basic underlying analogy between circuits and boolean algebra in response to the question 'Was the basic insight that yes/no can be embodied in on/off switches so trivial? ' with the following comments [17, p. xxvi]:

1000000000

It's not so much that a thing is "open" or "closed," the "yes" or "no" that you mentioned. The real point is that two things in series are described by the word "and" in logic, so you would say this "and" this, while two things in parallel are described by the word "or." The word "not" connects with the back contact of a relay rather than the front contact. There are contacts which close when you operate the relay, and there are other contacts which open, so the word "not" is related to that aspect of relays. All of these things together form a more complex connection between Boolean algebra, if you like, or symbolic logic, and relay circuits.

The people who had worked with relay circuits were, of course, aware of how to make these things. But they didn't have the mathematical apparatus of the Boolean algebra to work with them, and to do them efficiently. ...

They all knew the simple fact that if you had two contacts in series both had to be closed to make a connection through. Or if they are in parallel, if either one is closed the connection is made. They knew it in that sense, but they didn't write down equations with plus and times, where plus is like a parallel connection and times is like a series connection. ${ }^{9}$

\footnotetext{
${ }^{8}$ In this same interview [17, pp. xxv-xxvi], Shannon described the act of making the connection between Boolean algebra and a relay circuit as " not the main thing" and declared that "[t]he more important, harder part was working out the details, how to interleave the topology of the switching circuits, the way the contacts are connected up and so on, with the Boolean algebra expressions. Working that out was a lot of fun. I think I had more fun doing that than anything else in my life, creatively speaking."

${ }^{9}$ In the only two scholarly articles which he published on this subject $[14,16]$, Shannon focused on 'hindrance' or 'impedance' at a contact as the central physical characteristic, rather than 'flow' across the contact resulting from a
} 


\section{0}

In other words, the application of Boolean algebra to circuits provided an actual physical representation for the corresponding symbolic operations. Diagrams depicting the two types of connections and the corresponding operations are shown in Figure 1 below. An example of how to represent a more complicated circuit with an algebraic equation, based on an example from Shannon [16, p. 589], is shown in Figure 2.

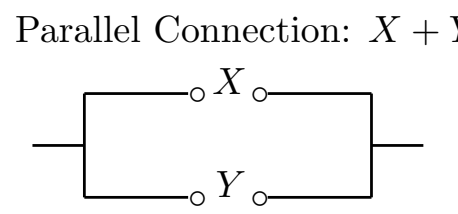

Series Connection: $X \cdot Y$

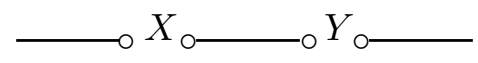

Figure 1

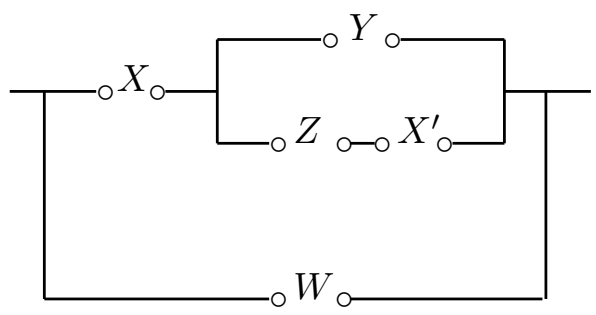

Figure 2: Network for $W+X \cdot\left(Y+Z \cdot X^{\prime}\right)$

1. Shannon employed the notation $X^{\prime}$ to represent the 'negative of $X$ ', or 'not- $X$ '; thus, the contact $X^{\prime}$ is closed (connection made between terminal points) whenever the contact $X$ is open (no connection made between terminal points), and vice versa. Explain why the overall network in Figure 2 is closed when contact $W$ is closed, regardless of the states of contacts $X$, $Y$ and $Z$. Then determine whether this network is open or closed when contact $W$ is open and contacts $X, Y$ and $Z$ are closed; explain your conclusion.

2. Represent the network in Figure 3 (adapted from [14, p. 715]) by a Boolean expression, using + for parallel connections and $\cdot$ series for connections.

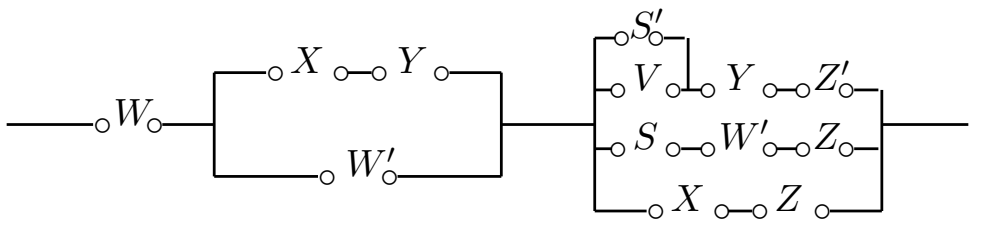

Figure 3: Network for project question 2.

connection being made. Under the impedance interpretation, an open circuit is said to have infinite impedance and a closed circuit is said to have zero impedance; in this interpretation, 'plus' corresponds to a series connection (infinite impedance when either the first or the second contact have infinite impedance: $a+b=1$ iff $a=1$ or $b=1$ ), while 'times' corresponds to a parallel connection (infinite impedance when the first and the second contact have infinite impedance: $a \cdot b=1$ iff $a=1$ and $b=1$ ). Owing to the dual principle of Boolean algebra, either interpretation (hindrance or flow) can be used with equal ease. In the interest of consistency with current textbook writing, we deviate from Shannon's original interpretation and base all exercises in this project on the 'flow' interpretation as described in Shannon's 1987 interview above; examples from Shannon's earlier papers are modified accordingly. 
3. Sketch the network represented by the Boolean expression $X+Y(Z+W)+X^{\prime} Z$, again using + for parallel connections and $\cdot$ series for connections.

Shannon used diagrams such as these not only to represent given circuits, but also to illustrate Boolean algebra identities. The following excerpt gives his description of the basic Boolean identities for circuits [14, p. 713-714].

$$
\begin{aligned}
& X+Y=Y+X \\
& X Y=Y X \\
& X+(Y+Z)=(Z+Y)+Z \\
& X(Y Z)=(X Y) Z \\
& X(Y+Z)=X Y+X Z \\
& X+Y Z=(X+Y)(X+Z) \\
& 1 \cdot X=X \\
& 0+X=X \\
& 1+X=1 \\
& 0 \cdot X=0
\end{aligned}
$$

Due to the associative laws ( $2 a$ and $2 b$ ) parentheses may be omitted in a sum or product of several terms without ambiguity. ...

The distributive law (3a) makes it possible to "multiply out" products and to factor sums. The dual of this theorem ( $3 b)$, however, is not true in numerical algebra.

... The negative of a hindrance $X$ will be written $X^{\prime}$ and is defined as a variable which is equal to 1 when $X$ equals 0 and equal to 0 when $X$ equals 1 . .. The definition of the negative of a hindrance gives the following theorems:

$$
\begin{aligned}
& X+X^{\prime}=1 \\
& X X^{\prime}=0 \\
& 0^{\prime}=1 \\
& 1^{\prime}=0 \\
& \left(X^{\prime}\right)^{\prime}=X
\end{aligned}
$$

\section{0}

Many of the laws listed above by Shannon are familiar from standard algebra. The most unfamiliar law, perhaps, is his (3b): $X+Y Z=(X+Y)(X+Z)$. The fact that (logical) addition is distributive over (logical) multiplication was, however, already a familiar boolean algebra property to Shannon's predecessors. The following question examines this law from the perspective of circuits.

4. Figure 4 below (adapted from [16, p. 591]) uses network diagrams to illustrate the distributive law (3b). Explain why these two circuits are equivalent by discussing what configurations of open (no connection made) and closed (connection is made) contacts are needed for flow across the overall network. 


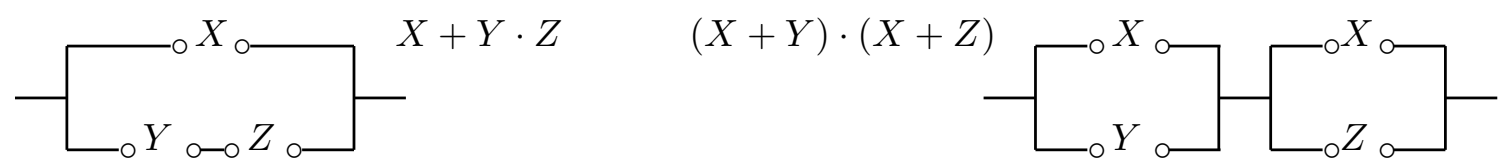

Figure 4: Network for project question 4.

Now complete the table below to show that the two Boolean expression are equivalent for all possible values of the variables in the boolean algebra on $\{0,1\}$.

\begin{tabular}{||c|c|c||c|c|c||c|c||}
\hline$X$ & $Y$ & $Z$ & $Y Z$ & $X+Y$ & $X+Z$ & $X+Y Z$ & $(X+Y)(X+Z)$ \\
\hline 0 & 0 & 0 & & & & & \\
\hline 0 & 0 & 1 & & & & & \\
\hline 0 & 1 & 0 & & & & & \\
\hline 0 & 1 & 1 & & & & & \\
\hline 1 & 0 & 0 & & & & & \\
\hline 1 & 0 & 1 & & & & & \\
\hline 1 & 1 & 0 & & & & & \\
\hline 1 & 1 & 1 & & & & & \\
\hline
\end{tabular}

Shannon referred to the proof technique in which all possible cases are directly verified, as is done in the above table, as the 'method of perfect induction' [14, p. 714]. In light of its simplicity, his 1938 paper included only one proof as an illustration of the technique. As Shannon himself noted, however, this proof technique is helpful in the context of circuits precisely because 'each variable is limited to just two values' [14, p. 714]. Since this is not the case for all boolean algebras, establishing these identities in general required more sophisticated proof techniques, such as those used in Huntington's 1904 paper on the axiomatization of boolean algebra. Because Shannon was interested in applying the properties of general boolean algebras to the specific two-valued boolean algebra defined by circuits, he proceeded to show that the two-valued algebra of circuits did, in fact, satisfy all Huntington's axioms for a general boolean algebra. As a consequence, any property which could be proven for a general boolean algebra necessarily held for the specific two-valued algebra of circuits.

Having established this correspondence, Shannon next listed several other boolean algebra identities, citing them as immediate consequences of the equivalence between circuits and symbolic logic. Among the more important of these were the following [14, pp. 714-715]:

10000000000

$$
\begin{aligned}
& (X+Y+Z+\ldots)^{\prime}=X^{\prime} \cdot Y^{\prime} \cdot Z^{\prime} \ldots \\
& (X \cdot Y \cdot Z \ldots)^{\prime}=X^{\prime}+Y^{\prime}+Z^{\prime} \ldots \\
& \\
& \ldots \ldots \ldots \ldots \ldots \ldots \ldots \ldots \ldots \ldots \ldots \\
& \\
& X=X+X=X+X+X=\text { etc. } \\
& X=X \cdot X=X \cdot X \cdot X=\text { etc. } \\
& X+X Y=X \\
& X(X+Y)=X
\end{aligned}
$$$$
\text { (9a) }
$$$$
\text { (9b) }
$$ 
Notice that, as he had already done with the first three postulates for circuits and with the basic identities (1) - (7), Shannon arranged these properties as pairs 'to emphasize a duality relationship between the operations of addition and multiplication and the quantities zero and one' [14, pp. 713]. This principle of duality was also well-known to his predecessors, and served as 'a characteristic feature of the algebra' [10, p. 294]. As noted by Shannon, this principle also 'gives each theorem a dual theorem, it being necessary to prove only one to establish the both.' [14, pp. 713]. Thus, as an immediate consequence of the Idempotent Law for Multiplication - Boole's $x^{2}=x$ and Shannon's Property (14b) — we are able to conclude that the Idempotent Law for Addition — Shannon's Property (14a) - is also valid. The following question explores the use of Shannon's method of perfect induction and the principle of duality within the context of Shannon's Identities (9ab) and (15ab), known as DeMorgan's Laws and Absorption respectively.

5. This question examines the Laws of Absorption, Shannon's Properties (15ab), in more detail.

(a) First complete the following tables for the two Absorption Laws, and comment on how they prove the validity of these two properties within the context of circuits.

\begin{tabular}{||l|l||l|l||}
\hline$X$ & $Y$ & $X Y$ & $X+X Y$ \\
\hline \hline 0 & 0 & & \\
\hline 0 & 1 & & \\
\hline 1 & 0 & & \\
\hline 1 & 1 & & \\
\hline
\end{tabular}

\begin{tabular}{||c|c||l|l||}
\hline$X$ & $Y$ & $X+Y$ & $X(X+Y)$ \\
\hline \hline 1 & 1 & & \\
\hline 1 & 0 & & \\
\hline 0 & 1 & & \\
\hline 0 & 0 & & \\
\hline
\end{tabular}

(b) Now compare these two tables, and comment on how they illustrate the ' duality relationship between the operations of addition and multiplication and the quantities zero and one'.

(c) Recall that in Boole's logic of classes multiplication corresponds to the operation of set intersection and addition corresponds to the operation of set union. Use this interpretation to explain why the Absorption Laws are also valid within this interpretation.

6. This question examines DeMorgan's Laws [Shannon's Properties (9ab)] in more detail.

(a) For two variables $X$ and $Y$, the first of DeMorgan's Law is the equality: $(X+Y)^{\prime}=X^{\prime} \cdot Y^{\prime}$ Complete the following tables to show that this law holds for circuits by the method of perfect induction.

\begin{tabular}{||c|c||c|c||c|c|c||}
\hline$X$ & $Y$ & $X+Y$ & $(X+Y)^{\prime}$ & $X^{\prime}$ & $Y^{\prime}$ & $X^{\prime} \cdot Y^{\prime}$ \\
\hline \hline 0 & 0 & & & & & \\
\hline 0 & 1 & & & & & \\
\hline 1 & 0 & & & & & \\
\hline 1 & 1 & & & & & \\
\hline
\end{tabular}

Then use the principle of duality to explain why the following dual version of DeMorgan's Law for two sets is also valid: $(X \cdot Y)^{\prime}=X^{\prime}+Y^{\prime}$.

(b) As stated by Shannon, DeMorgan's Law applied to any number of sets. Shannon also described this generalization as a (mechanical) method by which "the negative of any function may be obtained by replacing each variable by its negative and interchanging the + 
and $\cdot$ rules. ... For example, the negative of $X+Y \cdot\left(Z+W X^{\prime}\right)$ will be $X^{\prime}\left[Y^{\prime}+Z^{\prime}\left(W^{\prime}+X\right)\right]$ [14, p. 715]."

Without Shannon's method, negation of complicated expressions requires iterative applications of the basic two-set version of De Morgan's Laws: $(X Y)^{\prime}=X^{\prime}+Y^{\prime}$ and $(X+Y)^{\prime}=X^{\prime} Y^{\prime}$. For example, taking the example considered by Shannon above, we have:

$$
\begin{aligned}
{\left[X+Y \cdot\left(Z+W X^{\prime}\right)\right]^{\prime} } & =X^{\prime} \cdot \underbrace{\left[Y \cdot\left(Z+W X^{\prime}\right)\right]^{\prime}} \\
& =X^{\prime} \cdot[Y^{\prime}+\underbrace{\left(Z+W X^{\prime}\right)^{\prime}}] \\
& =X^{\prime} \cdot\left[Y^{\prime}+(Z^{\prime} \cdot \underbrace{\left(W X^{\prime}\right)^{\prime}})\right] \\
& =X^{\prime} \cdot\left[Y^{\prime}+Z^{\prime} \cdot\left(W^{\prime}+X^{\prime \prime}\right)\right] \\
& =X^{\prime} \cdot\left[Y^{\prime}+Z^{\prime} \cdot\left(W^{\prime}+X\right)\right]
\end{aligned}
$$

Re-write each of the following by first using Shannon's method, and then via the iterative De Morgan's Law technique. Which method do you prefer, and why?
(i) $\left[X Y^{\prime}+Z\right]^{\prime}$
(ii) $\left[X W\left(Y^{\prime}+Z\right)\right]^{\prime}$

(c) At one point in his work on general boolean algebras, Huntington showed how De Morgan's Laws can be used to define the operation + in terms of the operations - and '; namely, $X+Y=\left(X^{\prime} \cdot Y^{\prime}\right)^{\prime}$. Does this mean that all relay networks can be constructed using only series connections? Explain.

One of Shannon's goals in applying boolean algebra to the study of circuits was to use algebraic techniques to simplify complicated systems, as he described in the following excerpt from his 1949 paper The Synthesis of Two-Terminal Switching Circuits [16, p. 590].

000000000

By means of Boolean Algebra it is possible to find many circuits equivalent in operating characteristics to a given circuit. The hindrance of the given circuit is written down and manipulated according to the rules. Each different resulting expression represents a new circuit equivalent to the given one. In particular, expressions may be manipulated to eliminate elements which are unnecessary, resulting in simple circuits.

\section{0}

For example, the following computation confirms that the element $Z$ can be eliminated from the network from Figure 2, a fact which careful examination of the network diagram in Figure 2 also reveals:

$W+X \cdot\left(Y+Z \cdot X^{\prime}\right)=W+X Y+X\left(Z X^{\prime}\right)=W+X Y+\left(X X^{\prime}\right) Z=W+X Y+0 \cdot Z=W+X Y$ 


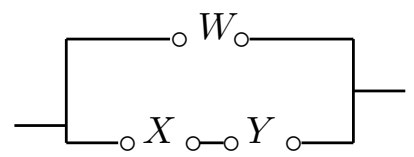

Figure 5: A network equivalent for $W+X\left(Y+Z \cdot X^{\prime}\right)$.

7. Use boolean algebra identities, including the law of absorption (Shannon's properties 15ab) to show that the networks in Figure 6 are equivalent.

(a)

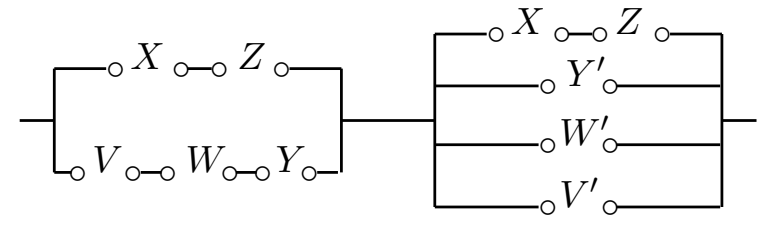

(b)

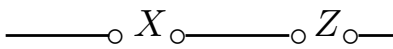

Figure 6: Networks for project question 7.

8. Use boolean algebra identities to show that the network represented by the expression $W$. $\left(X Y+W^{\prime}\right) \cdot\left[S^{\prime} Y Z^{\prime}+V Y Z^{\prime}+S W Z+X Z\right]$ is equivalent to the network in Figure 7. Then sketch the network for the full expression $W \cdot\left(X Y+W^{\prime}\right) \cdot\left[S^{\prime} Y Z^{\prime}+V Y Z^{\prime}+S W Z+X Z\right]$, and comment on the relative simplicity of the two equivalent networks.

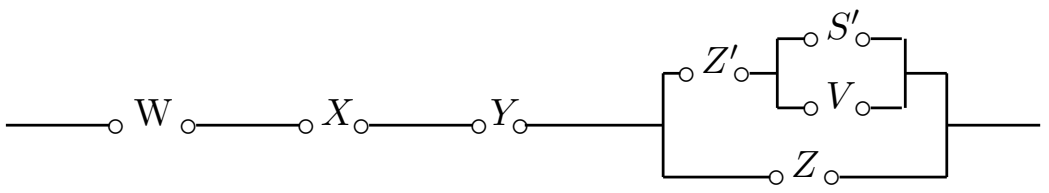

Figure 7: Network for project question 8.

To algebraically simplify very complicated networks, such as that in Figure 3 above, Shannon employed a method of representing Boolean-valued functions dating back to Boole's own Laws of Thought [5]. In fact, this method of representation formed the core of Boole's technique of logical deduction via algebraic manipulation. Like Boole, Shannon compared this method to a familiar idea from calculus [14, p. 715].

1000000000

The notation $f\left(X_{1}, X_{2}, \ldots X_{n}\right)$ will be used to represent a function. Thus, we have $F(X, Y, Z)=X Y+X^{\prime}\left(Y^{\prime}+Z^{\prime}\right)$. In infinitesimal calculus it is shown that any function (providing it is continuous and all derivatives are continuous) may be expanded in a Taylor series. A somewhat similar expansion is possible in the calculus of propositions. To develop the series expansion of functions first note the following equations. 


$$
\begin{aligned}
& f\left(X_{1}, X_{2}, \ldots X_{n}\right)=X_{1} \cdot f\left(1, X_{2}, \ldots X_{n}\right)+X_{1}^{\prime} f\left(0, X_{2}, \ldots X_{n}\right) \\
& f\left(X_{1}, X_{2}, \ldots X_{n}\right)=\left[f\left(0, X_{2}, \ldots X_{n}\right)+X_{1}\right] \cdot\left[f\left(1, X_{2}, \ldots X_{n}\right)+X_{1}^{\prime}\right]
\end{aligned}
$$

These reduce to identities if we let $X_{1}$ equal either 0 or 1 . In these equations the function $f$ is said to be expanded about $X_{1}$.

Some other theorems useful in simplifying expressions are given below:

$$
\begin{aligned}
& X f(X, Y, \ldots Z)=X f(1, Y, \ldots Z) \\
& X+f(X, Y, \ldots Z)=X+f(0, Y, \ldots Z)
\end{aligned}
$$

All of these theorems may be proved by the method of perfect induction.

\section{0}

9. Verify that Shannon's identity $10 a$ using his method of perfect induction.

That is, first show that this identity holds in the case $X_{1}=0$, simplifying the right-hand side as needed, and then verify that this identity holds in the case $X_{1}=1$.

Then provide similar proofs for Shannon's identities $10 b, 17 a$ and $17 b$.

10. In his work on the logic of classes, Boole represented $x^{\prime}$ by the expression $1-x$ (i.e., the universal set ' 1 ' with the set ' $x$ ' removed). Writing $f(x)=a x+b(1-x)$, he was therefore able to algebraically solve for the coefficients $a$ and $b$ simply by letting $x=0$ and $x=1$ respectively $[5$, p. 72]. Verify that this gives the expansion $f(x)=f(1) x+f(0)(1-x)$, and compare this expression to Shannon's identity $10 a$.

We will consider Shannon's expansion of a function about two or more variables in Section 3 of this project. First, we look at how he used the expansion about one variable given in identity $17 \mathrm{~b}$ to simplify circuits $[14, \text { p. } 715]^{10}$.

10000000000

The hindrance function $X_{a b}$ for this circuit will be:

$$
\begin{aligned}
X_{a b} & =W+W^{\prime}(X+Y)+(X+Z)\left(S+W^{\prime}+Z\right)\left(Z^{\prime}+Y+S^{\prime} V\right) \\
& =W+X+Y+(X+Z)(S+1+Z)\left(Z^{\prime}+Y+S^{\prime} V\right) \\
& =W+X+Y+Z \cdot\left(Z^{\prime}+Y+S^{\prime} V\right)
\end{aligned}
$$

These reductions were made with $17 \mathrm{~b}$ using first $W$, then $X$ and $Y$ as the " $X$ " of $17 \mathrm{~b}$. Now multiplying out

$$
\begin{aligned}
X_{a b} & =W+X+Y+Z Z^{\prime}+Z Y+Z S^{\prime} V \\
& =W+X+Y+Z S^{\prime} V
\end{aligned}
$$

\footnotetext{
${ }^{10}$ Note that this expression is the dual of the expression for the circuit shown in Figure 3 above.
} 
11. This question provides a slightly different derivation of Shannon's simplified form for $X_{a b}$ from the preceding excerpt.

Begin by letting

$$
f(W, X, Y, Z, S, V)=W^{\prime}(X+Y)+(X+Z)\left(S+W^{\prime}+Z\right)\left(Z^{\prime}+Y+S^{\prime} V\right),
$$

so that $X_{a b}=W+f(W, X, Y, Z, S, V)$.

Explain why

$$
f(0, X, Y, Z, S, V)=X+Y+(X+Z)(S+1+Z)\left(Z^{\prime}+Y+S^{\prime} V\right) .
$$

Why can we replace this by last expression by $X+Y+(X+Z)\left(Z^{\prime}+Y+S^{\prime} V\right)$ ?

Using this latter value for $f(0, X, Y, Z, S, V)$ in identity $17 b$, conclude that

$$
X_{a b}=W+f(W, X, Y, Z, S, V)=W+X+Y+(X+Z)\left(Z^{\prime}+Y+S^{\prime} V\right) .
$$

(Up to this point, our derivation corresponds essentially Shannon's first step.)

Next, let $g(X, Y, Z, S, V)=Y+(X+Z)\left(Z^{\prime}+Y+S^{\prime} V\right)$, so that $X_{a b}=W+[X+g(X, Y, Z, S, V)]$. Explain why $g(0, Y, Z, S, V)=Y+Y X^{\prime} V$, then use this value in identity $17 b$ to conclude that

$$
X_{a b}=W+X+Y+Z S^{\prime} V
$$

12. The derivation outlined in project question 11, as well as Shannon's derivation of that same simplified form, both made use of Shannon's identity 17b. Try to obtain this same simplified form by instead using the more standard algebraic process of expansion (i.e., using distributivity of multiplication over addition), beginning with the original expression $W+W^{\prime}(X+Y)+(X+$ $Z)\left(S+W^{\prime}+Z\right)\left(Z^{\prime}+Y+S^{\prime} V\right)$. Comment on the efficiency of these different approaches to simplification.

\section{Boolean Functions and Synthesis of Circuits}

In 1892, a supporter of Boole's approach to logic, W. E. Johnson, wrote [11, p. 3]:

As a material machine is an instrument for economising the exertion of force, so a symbolic calculus is an instrument for economising the exertion of intelligence. And, employing the same analogy, the more perfect the calculus, the smaller would be the amount of intelligence applied as compared with the results produced. ... It will appear that the logical calculus stands in a unique relation to intelligence; for it aims at exhibiting, in a non-intelligent form those same intelligent principles that are actually required for working it.

The circuits used in modern computing technology also serve as an economizing instrument for the exertion of both force and intelligence, with Boole's logical calculus providing the necessary nonintelligent, mechanical mode for making them work in required ways. In this closing section of this project, we consider the problem of "synthesis" for circuits: given a specific set of desired inputs and outputs, construct a network of series and parallel connections corresponding to those values. 
The mathematical ideas needed to solve this problem were, in fact, developed by Boole in connection with problems in logic. Boole's goal was to take a logical expression that involved any number of logical variables and represent it as a particular kind of sum. For a logical expression $f(x)$ of just one variable, the desired sum had the form $f(x)=a x+b(1-x)$. Boole referred to this process as "developing $f(x)$ ". The following excerpt shows how Boole developed a method for computing the coefficients $a$ and $b$ in this sum [5, p. 74].

1000000000

Assume then,

$$
f(x)=a x+b(1-x),
$$

and making $x=1$, we have

$$
f(1)=a .
$$

Again, in the same equation making $x=0$, we have

$$
f(0)=b \text {. }
$$

Hence, the values of $a$ and $b$ are determined, and substituting them in the first equation, we have

$$
f(x)=f(1) x+f(0)(1-x) ;
$$

as the development sought ${ }^{11}$.

1000000000

Using Shannon's notation $x^{\prime}$ to denote the expression $1-x$, note that this equation can be re-written as:

$$
f(x)=f(1) x+f(0) x^{\prime} .
$$

Before looking at a specific example, read Boole's description of how to develop a function $f(x, y)$ of two variables $[5$, p. 74$]$ :

1000000000

... we have

$$
f(x, y)=f(1,1) x y+f(1,0) x(1-y)+f(0,1)(1-x) y+f(0,0)(1-x)(1-y),
$$

for the expansion required. Here $f(1,1)$ represents what $f(x, y)$ becomes when we make therein $x=1, y=1 ; f(1,0)$ represents what $f(x, y)$ becomes when we make therein $x=1$, $y=0$, and so on for the rest.

\section{0}

\footnotetext{
${ }^{11}$ Boole included a footnote at this point in which he showed how to derive this same equation by substituting $x^{n}=x$ into the Taylor's series expansion of a function $f(x)$ and manipulating the result algebraically.
} 
13. Using Shannon's notation of $x^{\prime}$ for $1-x$, Boole's expansion of $f(x, y)$ can be written as

$$
f(x, y)=f(1,1) x y+f(1,0) x y^{\prime}+f(0,1) x^{\prime} y+f(0,0) x^{\prime} y^{\prime} .
$$

Use this expansion to verify that the following table of function values defines the function represented by $f(x, y)=x y+x^{\prime} y$.

\begin{tabular}{||c|c||c||}
\hline$x$ & $y$ & $f(x, y)$ \\
\hline 0 & 0 & 0 \\
\hline 0 & 1 & 1 \\
\hline 1 & 0 & 0 \\
\hline 1 & 1 & 1 \\
\hline
\end{tabular}

14. Use Boole's notation to write out the eight terms of the expansion for a function of three variables, $f(x, y, z)$; then translate this into Shannon's notation.

Shannon also considered this type of expansion for a function $f\left(x_{1}, x_{2}, \ldots, x_{n}\right)$ of $n$ variables. In both $[14,16]$, he noted that the expansion will include $2^{n}$ terms, where each of these term will have the form ' $C y_{1} y_{2} y_{3} \ldots y_{n}$ ' with the coefficient $C$ equal to either 0 or 1 and each $y_{i}$ equal to either $x_{i}$ or $x_{i}^{\prime}$. Today, this form of expansion is referred to as the disjunctive normal form of the function.

As an example, note that the function $f(x, y, z)=x^{\prime} y+y^{\prime} z^{\prime}+x y z$ is NOT in disjunctive normal form as it is currently written, since the variable $z$ is missing from the first term, while the variable $x$ is missing from the second term. However, we can re-write this function in disjunctive normal form as follows: $f(x, y, z)=x^{\prime} y z+x^{\prime} y z^{\prime}+x y^{\prime} z^{\prime}+x^{\prime} y^{\prime} z^{\prime}+x y z$. Notice that some of the terms in the disjunctive normal form have zero coefficients, and therefore do not need to be written.

The next project question illustrates two methods for finding the disjunctive normal form of any function, given the function as a boolean expression.

15. Consider the function $f(x, y, z)=y z+x y^{\prime}$.

(a) Calculate the values of $f(x, y, z)$ for all possible values of $x, y, z$, and use these values in your expansion from project question 14 to find the disjunctive normal form of $f$. (You should find four non-zero terms.)

(b) Now use the facts that $x+x^{\prime}=1$ and $z+z^{\prime}=1$ to find the disjunctive normal form by expanding $f(x, y, z)=1 \cdot(y z)+1 \cdot\left(x y^{\prime}\right)$.

(c) Which method do you prefer, and why?

The remaining project question illustrate the use of the disjunctive normal form to find a boolean expression of any function, given the function as a table of values. 
16. Suppose we wish to build a circuit which corresponds to the following table of values for an unknown function $f(x, y, z)$ given below.

\begin{tabular}{||c|c|c||c||}
\hline$x$ & $y$ & $z$ & $f(x, y, z)$ \\
\hline 0 & 0 & 0 & 0 \\
\hline 0 & 0 & 1 & 0 \\
\hline 0 & 1 & 0 & 1 \\
\hline 0 & 1 & 1 & 1 \\
\hline 1 & 0 & 0 & 1 \\
\hline 1 & 0 & 1 & 0 \\
\hline 1 & 1 & 0 & 1 \\
\hline 1 & 1 & 1 & 1 \\
\hline
\end{tabular}

Use your expansion from project question 14 to find the disjunctive normal form of $f$, and simplify your result to show that $f(x, y, z)=y+x z^{\prime}$. Then sketch the network corresponding to $y+x z^{\prime}$. How easy would it have been to determine this circuit directly from the table of values, rather than from its Boolean expression?

17. Find the disjunctive normal form of the function $f$ represented by the following table, and use it to sketch the corresponding network.

\begin{tabular}{||c|c|c||c||}
\hline$x$ & $y$ & $z$ & $f(x, y, z)$ \\
\hline 0 & 0 & 0 & 1 \\
\hline 0 & 0 & 1 & 0 \\
\hline 0 & 1 & 0 & 0 \\
\hline 0 & 1 & 1 & 1 \\
\hline 1 & 0 & 0 & 0 \\
\hline 1 & 0 & 1 & 0 \\
\hline 1 & 1 & 0 & 0 \\
\hline 1 & 1 & 1 & 1 \\
\hline
\end{tabular}

18. Find the disjunctive normal form of the function $f$ represented by the following table, and use it to sketch the corresponding network.

\begin{tabular}{||c|c|c||c||}
\hline$x$ & $y$ & $z$ & $f(x, y, z)$ \\
\hline 0 & 0 & 0 & 0 \\
\hline 0 & 0 & 1 & 0 \\
\hline 0 & 1 & 0 & 0 \\
\hline 0 & 1 & 1 & 1 \\
\hline 1 & 0 & 0 & 1 \\
\hline 1 & 0 & 1 & 1 \\
\hline 1 & 1 & 0 & 1 \\
\hline 1 & 1 & 1 & 0 \\
\hline
\end{tabular}

19. Describe a general method for finding the disjunctive normal form of a Boolean function $f$ from its table of values.

20. How would you define the conjunctive normal form of a Boolean function? Give an example, and discuss possible method(s) for finding this form for a given function in disjunctive normal form and/or from a table of values. 


\section{Notes to the Instructor}

This project is designed for an introductory or intermediate course in discrete or finite mathematics that considers boolean algebra from either a mathematical or computer science perspective. The project does assume some (very minimal) familiarity with the set operations of union and intersection. This pre-requisite material may be gained by completing the companion (Boole) project described below, through reading a standard textbook treatment of elementary set operations, or via a short class discussion/lecture.

Based on a award-winning paper by Claude Shannon, A Symbolic Analysis of Relay and Switching Circuits, this project begins with a concise overview of two historical antecedents to Shannon's work. The first of these is George Boole's original work on 'the logic of classes,' included in part to provide students with a connection to another concrete example of a boolean algebra on which they can draw; the second of these is Edward Huntington's work on the axiomatization of boolean algebras, included in part to emphasize to students the relationship between abstract axiomatic structures and concrete models as examples of those structures. Section 2 of the project introduces and develops the use of boolean expressions to represent parallel and series circuits. Within the concrete context of the 2-valued boolean algebra associated with these circuits, the standard properties of a boolean algebra are developed in this section; specific project questions in this section also provide students with practice in using these identities to simplify and manipulate boolean expressions. In Section 3 , the concept of a 'disjunctive normal form' for boolean expressions is introduced in the context of circuit design.

Two other projects on boolean algebra are available as companions to this project, either or both of which could also be used independently of this project. The first companion project "Origins of Boolean Algebra in the Logic of Classes: George Boole, John Venn and C. S. Peirce," is suitable as a preliminary to either the Huntington project or to the Shannon project. Without explicitly introducing modern notation for operations on sets (until the concluding section), that project develops a modern understanding of these operations and their basic properties within the context of early efforts to develop a symbolic algebra for logic. By steadily increasing the level of abstraction, that project also lays the ground work for a more abstract discussion of boolean algebra as a discrete structure, and explores a variety of other mathematical themes, including the notion of an inverse operation, issues related to mathematical notation, and standards of rigor and proof.

The second companion project "Boolean Algebra as an Abstract Structure: Edward V. Huntington and Axiomatization" could be used either as a preliminary to or as a follow-up to the Shannon project on circuit design. That project explores the early axiomatization of boolean algebra as an abstract structure, based on Huntington's 1904 paper Sets of Independent Postulates for the Algebra of Logic. In addition to introducing the now standard axioms for the boolean algebra structure, the project illustrates how to use these postulates to prove some basic properties of boolean algebras. Specific project questions also provide students with practice in using symbolic notation, and encourage them to analyze the logical structure of quantified statements. The project also examines Huntington's use of the two-valued Boolean algebra on $K=\{0,1\}$ - first studied by George Boole in his work on the logic of classes - as a model to establish the independence and consistency of one of his postulate sets. The final section of the project discusses modern (undergraduate) notation and axioms for boolean algebras, and provides several practice exercises to reinforce the ideas developed in the earlier sections.

Implementation with students of any of these projects may be accomplished through individually assigned work, small group work and/or whole class discussion; a combination of these instructional strategies is recommended in order to take advantage of the variety of questions included in the project. 


\section{References}

[1] Bocheński, I. M., A History of Formal Logic, Thomas, I. (translator \& editor), University of Notre Dame Press, Notre Dame, 1961.

[2] Boole, G., Mathematical Analysis of Logic, MacMillan, Barclay \& MacMillan, Cambridge, 1847.

[3] Boole, G., Mathematical Analysis of Logic, Open Court, La Salle, 1952.

[4] Boole, G., An Investigation of the Laws of Thought on Which are Founded the Mathematical Theories of Logic and Probabilities, Walton and Maberly, London, 1854.

[5] Boole, G., An Investigation of the Laws of Thought on Which are Founded the Mathematical Theories of Logic and Probabilities, Dover Publications, , New York, 1958.

[6] Couturat, C., The Algebra of Logic, Robinson, L. G. (translator), Open Court, Chicago, 1914.

[7] De Morgan, A., Formal Logic: or, The Calculus of Inference, Necessary and Probable, Taylor and Walton, London, 1847.

[8] De Morgan, A., Trigonometry and Double Algebra, Taylor, Walton 8 Maberly, London, 1849.

[9] Gillispie, C. C., Holmes, F.L., (editors) Dictionary of Scientific Biography, Charles Scribner's Sons, New York, 1970.

[10] Huntington, E. V., Sets of Independent Postulates for the Algebra of Logic, Transactions of the American Mathematical Society, 5:3 (1904), 288-309.

[11] Johnson, W. E., The Logic Calculus. I. General Principles, Mind, 1:1 (1892), 3-30.

[12] Lewis, C. I., A Survey of Symbolic Logic: The Classic Algebra of Logic, Dover Publications, New York, 1960.

[13] Merrill, D., Augustus De Morgan and the Logic of Relations, Kluwer, Dordrecht, 1990.

[14] Shannon, C. E., A Symbolic Analysis of Relay and Switching Circuits, American Institute of Electrical Engineers Transactions, 57 (1938), 713-723. Reprinted in [17], 471-495.

[15] Shannon, C.E., "A Mathematical Theory of Communication," Bell System Technical Journal, 27 (1948), 379-423 and 623-656.

[16] Shannon, C. E., The Synthesis of Two-Terminal Switching Circuits, Bell System Technical Journal, 28 (1949), 417-425. Reprinted in [17], 588-627.

[17] Sloane, N. J. A. \& Wyner, A. D. (editors), Claude Elwood Shannon: Collected Papers, IEEE Press, New York, 1993.

[18] Smith, G. C., The Boole-DeMorgan Correspondence, 1842-1864, Clarendon Press, Oxford, 1982.

[19] Venn, J., Symbolic Logic, MacMillan, London, 1881.

[20] Venn, J., Symbolic Logic, MacMillan, London, 1894. Reprint Chelsea, Bronx 1971.

[21] Whitesitt, J. E., Boolean Algebra and Its Applications,, Addison-Wesley, Reading, 1961. Reprinted by Dover Publications, New York, 1995. 